\title{
Thrombopoietin receptor agonist therapy in primary immune thrombocytopenia is associated with bone marrow hypercellularity and mild reticulin fibrosis but not other stromal abnormalities
}

\author{
Leonardo Boiocchi ${ }^{1,2}$, Attilio Orazi ${ }^{1}$, Waleed Ghanima ${ }^{3,4}$, Melissa Arabadjief ${ }^{1}$,
} James B Bussel ${ }^{3}$ and Julia Turbiner Geyer ${ }^{1}$

${ }^{1}$ Department of Pathology and Laboratory Medicine, Weill Cornell Medical College, New York, NY, USA; ${ }^{2}$ Pathology Unit, Department of Medicine, Surgery and Dentistry, University of Milan Medical School, Fondazione IRCCS Ca' Granda-Ospedale Maggiore Policlinico, Milan, Italy; ${ }^{3}$ Department of Pediatrics, Weill Cornell Medical College, New York, NY, USA and ${ }^{4}$ Department of Medicine, Østfold Hospital Trust, Fredrikstad, Norway

\begin{abstract}
Primary immune thrombocytopenia is an acquired autoimmune disorder characterized by platelet count of $<100 \times 10^{9} / \mathrm{l}$ in the absence of other causes of thrombocytopenia. Primary immune thrombocytopenia is defined as 'chronic' when it has been present for more than $\mathbf{1 2}$ months without spontaneous remission or maintenance of complete response to therapy. Recently, thrombopoietin receptor agonists became available for treatment of chronic primary immune thrombocytopenia. Anecdotal reports have raised concerns about a possible association between therapy with thrombopoietin receptor agonists and an increase in bone marrow fibrosis. To investigate this association we studied eight patients with primary immune thrombocytopenia in detail comparing fibrosis and other morphological features in pre-therapy and on-therapy bone marrow biopsies, with the longest follow-up reported to date. A slight but significant increase to MF-1 in reticulin fibrosis was observed during therapy, but collagen was never present. On-therapy bone marrows were hypercellular due to panmyelosis with increased trilineage hematopoiesis. Megakaryocytes were increased in number, with acquisition of evident pleomorphism, nuclear hyperlobulation and tendency in some cases to form clusters. The overall picture of the on-therapy marrows was characterized by myeloproliferative neoplasm-like features, resembling essential thrombocythemia or occasionally early primary myelofibrosis. As thrombopoietin receptor agonists are becoming a mainstream treatment for primary immune thrombocytopenia, general pathologists and especially hematopathologists need to be aware of the characteristic morphological changes associated with use of these therapeutic agents, in order to avoid misdiagnosis of a myeloid neoplasm.
\end{abstract}

Modern Pathology (2012) 25, 65-74; doi:10.1038/modpathol.2011.128; published online 12 August 2011

Keywords: bone marrow biopsy; c-MPL; megakaryocyte; myeloproliferative marrow; primary immune thrombocytopenia; thrombopoietin receptor agonists

Primary immune thrombocytopenia is an acquired autoimmune disorder characterized by isolated thrombocytopenia (defined as a peripheral blood

Correspondence: Dr JT Geyer, MD, Department of Pathology and Laboratory Medicine, Weill Cornell Medical College, 525 East 68th Street, New York, NY 10065, USA.

E-mail: jut9021@med.cornell.edu

Received 2 May 2011; revised 23 June 2011; accepted 23 June 2011; published online 12 August 2011 platelet count $<100 \times 10^{9} / \mathrm{l}$ ) in the absence of other causes or disorders that may be associated with thrombocytopenia. The decrease in the platelet count in primary immune thrombocytopenia is mediated by autoantibodies directed against platelets and megakaryocytes, causing an accelerated clearance of platelets, inhibition in platelet production and megakaryocyte apoptosis. Multiple different immunological mechanisms, still not fully characterized, have been associated with the 
anti-platelet auto-antibodies. Despite the oftenmarked thrombocytopenia in patients with primary immune thrombocytopenia, thrombopoietin levels are at most slightly increased.

An increased risk of bleeding is the main clinical problem in primary immune thrombocytopenia, although bleeding symptoms may not always be present. Primary immune thrombocytopenia is defined as 'chronic' when it has been present for more than 12 months. ${ }^{1}$ The diagnosis remains one of exclusion; there is no specific clinical or laboratory diagnostic test available. Bone marrow examination, which is often part of the clinical assessment of primary immune thrombocytopenia, is primarily used to exclude other possible causes of thrombocytopenia and in particular to rule out myelodysplastic syndrome or a marrow failure state. It may also be performed during follow-up to evaluate causes of refractoriness to therapy.

In patients with primary immune thrombocytopenia, when the risk for haemorrhagic morbidity is high or the patient's quality of life is affected, therapy is initiated. First-line treatment includes glucocorticoids, intravenous immunoglobulin and/ or intravenous anti-D. In chronic primary immune thrombocytopenia the gold-standard treatment for patients who fail steroids is still splenectomy; ${ }^{2,3}$ rituximab can be used as an alternative to splenectomy, but less than half of treated patients achieve a sustained remission. ${ }^{4-6}$ The exact order and timing of treatments remains to be determined and is individualised for each patient according to the preferences of the doctor and patient.

The latest family of drugs that became available for the treatment of primary immune thrombocytopenia is the thrombopoietin receptor agonists. In 2008, two drugs of this family, romiplostim and eltrombopag, were approved for clinical use in the USA and in 2009 in Europe and increasingly elsewhere. Thrombopoietin receptor agonists bind and activate c-MPL, the thrombopoietin receptor on megakaryocytes and their precursors. ${ }^{7,8} \mathrm{C}-\mathrm{MPL}$ is a dimeric cytokine receptor that enhances survival and proliferation of megakaryocytes, leading to platelet production following binding to thrombopoietin. ${ }^{9-11}$

Bone marrow fibrosis has been episodically reported to occur in clinical trials following use of thrombopoietin receptor agonists. In one of these studies an increase in bone marrow reticulin was seen in five patients during evaluation of long-term treatment with romiplostim. These observations raised concerns about the safety of these drugs although, in the absence of other than anecdotal information, fibrosis is thought to be reversible when therapy is suspended. ${ }^{12,13}$

We sought to evaluate and characterize the morphological changes following treatment with thrombopoietin receptor agonists in patients with primary immune thrombocytopenia, by comparing their pre-treatment and post-treatment bone marrow biopsies. The expression of markers known to be correlated with the presence of fibrosis in myeloproliferative neoplasms was evaluated. ${ }^{14}$

\section{Materials and methods}

\section{Patients}

Paraffin-embedded bone marrow biopsies and bone marrow aspirates were obtained from the posterior superior iliac spine of eight patients with primary immune thrombocytopenia selected from the files of the Department of Pathology and Laboratory Medicine, Weill Cornell Medical College/New York Presbyterian Hospital (New York, NY, USA). The design of this study was approved by the institutional review board (IRB) of Weill Cornell Medical College. For each patient, two sets of bone marrow samples were examined, the first biopsy taken before starting the treatment with thrombopoietin receptor agonists, and the second one obtained during treatment. All bone marrow biopsies were fixed in Bouin's solution and acid decalcified. Sections were stained with hematoxylin and eosin (H\&E) for morphological examination; Gomori's silver impregnation and trichrome staining were used to evaluate fibrosis. The results of cytogenetic and FISH studies were available for all patients.

\section{Histochemistry}

Gomori's silver impregnation and trichrome histochemical stains were performed by an automated stainer (Leica Microsystems, Bannockburn, IL, USA), using manufacturer's specific kits and protocols, with only minor modifications.

\section{Immunohistochemistry}

Immunohistochemical studies were performed using a Bond-Max autostainer (Vision BioSystems, Hingham, MA, USA) using a polymer-defined peroxidase detection system after antigen retrieval with Epitope Retrieval Solution 2 (Vision BioSystems). Monoclonal antibodies against the following antigens were used: collagen type IV (CIV 22; Dako North America, Carpinteria, CA, USA), smooth muscle actin (1A4, Dako), low-affinity nerve growth factor receptor (ME20.4; Dako) and laminin (LAM89; Vision BioSystems Novocastra, Newcastle-upon-Tyne, UK).

\section{Morphological Analysis}

Histological evaluation was performed by three pathologists (LB, AO and JG) who were not aware whether the biopsy was taken before or during therapy. Discordant results were discussed until consensus was reached.

The evaluation of morphological features was performed on the H\&E sections. The following 
parameters were examined: (a) the absolute bone marrow cellularity; (b) the cellularity in relationship with the patient's age; (c) the quantity of erythropoiesis, granulopoiesis and megakaryopoiesis; and (d) the presence or absence of left-shifted erythropoiesis and granulopoiesis, defined as an increased number of immature precursors. The number of megakaryocytes was scored semiquantitatively as follows: 0, normal; $1+$, slightly increased (up to two times the normal value); $2+$, moderate increase (3-4 times the normal value); or $3+$, marked increase ( $>4$ times the normal value). ${ }^{15,16}$ Bone marrow cellularity in relationship with the expected cellularity for patient's age was scored as decreased, normal, increased or markedly increased $(0-3+)$.

In addition, we evaluated the presence or the absence of the following megakaryocyte features: (a) clusters as aggregates of at least three megakaryocytes, with or without the interposition of other marrow cells among megakaryocytes; (b) pleomorphism, defined as the presence of significant size variation; (c) maturation defects, defined as deviation in the nuclear/cytoplasmic ratio; atypical, hyperchromatic nuclei; and dense, eosinophilic cytoplasm; (d) nuclear hyperlobulation, defined as deep lobulation of the nuclei; (e) bulbous nuclei, defined as hypolobulation of the nuclei with chromatin clumping and a 'cloud-like' appearance; and (f) bare/naked megakaryocytic nuclei. ${ }^{17}$

\section{Evaluation of Fibrosis}

The degree of fibrosis in the bone marrow was evaluated using Gomori's (reactive with all types of fibres) and trichrome stainings (reactive with collagen fibres). A scale $0-3+$ (MF-0-MF-3) was used to grade reticulin fibrosis, according to the European Consensus Method. ${ }^{18}$ Collagen fibrosis was evaluated as negative or positive.

\section{Immunohistochemical Evaluation of the Extracellular Matrix and of Stromal Cell Populations}

Expression of type IV collagen and laminin was assessed in order to evaluate the extracellular matrix. Immunohistochemical stains for low-affinity nerve growth factor receptor were performed to identify the adventitial reticular cells, whereas smooth muscle actin stain identified myofibroblastic differentiation within the bone marrow stromal cells. ${ }^{14}$ The positivity was evaluated semi-quantitatively as negative $(0)$, focally positive $(1+)$, positive $(2+)$ or strongly diffusely positive $(3+)$.

\section{Statistical Analysis}

Statistical analysis of results was performed with the SPSS statistics 17 software (SPSS, Chicago, IL, USA). For all statistical tests a $P$-value $<0.05$ was considered significant; all tests were two-sided. Wilcoxon-signed rank test was used to evaluate differences in reticulin, in age-adjusted cellularity, in the number of megakaryocytes, and in the immunohistochemical extracellular matrix and stromal cells markers expression, before and after the start of the therapy. McNemar test was used to evaluate differences in the presence of clusters, maturational defects and bulbous or naked nuclei in megakaryocytes. A paired-samples $t$-test was applied to evaluate differences in the absolute cellularity and in the myeloid/erythroid ratio.

\section{Results}

\section{Clinical Features}

Clinical information was available for all patients at baseline, ie at the time of the biopsy preceding the therapy with thrombopoietin receptor agonists, at the time of initiation of therapy and at time of the on-therapy biopsy. The series included eight patients (three males and five females). The mean age at baseline was 40 years (range: 5-70 years) and was 44 years (range: 13-72 years) at the time of the on-therapy biopsy. All patients had received several cycles of different treatments before starting thrombopoietin receptor agonists therapy, and six had undergone splenectomy.

The mean duration of follow-up from baseline to the on-therapy marrow examination was 4.1 years (range: 0.7-7.9 years); the mean duration of followup from thrombopoietin receptor agonists treatment initiation to on-therapy biopsy was 2.7 years (range: 0.2-6.1years). The mean platelet value at baseline was $17 \times 10^{9} / \mathrm{l}$, whereas at the time of the follow-up marrow it was $105 \times 10^{9} / \mathrm{l}$. For the duration of this study, thrombopoietin receptor agonists were the only treatment used. All patients responded to therapy, and elevation in the number of platelets was the only haematological variation observed. Haemoglobin levels and white blood cell count (WBC) did not change. None of the patients had past medical history of any other condition known to be associated with marrow fibrosis. Cytogenetic analysis results of pre- and on-therapy marrow aspirates showed no abnormalities in all patients. Demographical and clinical data for each patient are summarised in Table 1.

\section{Morphological Evaluation}

The absolute cellularity was increased in all patients after starting thrombopoietin receptor agonists, compared with the corresponding cellularity observed in baseline biopsy $(P=0.03)$. Cellularity in relationship with patient's age was also increased in seven of eight patients $(88 \% ; P<0.01)$. The number of megakaryocytes observed in the on-treatment biopsy was higher than that at baseline 
in five of eight patients $(63 \%)(P=0.04)$ (Figure 1). In the remaining three patients the number of megakaryocytes was already elevated at baseline, and did not show a quantitative change in the on-therapy biopsy (Table 2). During therapy the megakaryocytes acquired marked nuclear hyperlobulation $(P=0.01)$ and showed a significant polymorphism with presence of many giant forms in all cases. In three cases, in the on-therapy biopsy these features were so striking that the megakaryocytes strongly resembled those typically seen in essential thrombocythemia. In addition, the on-therapy marrows showed a clear tendency for megakaryocytes to form clusters, closely resembling features seen in myeloproliferative neoplasms such as early primary myelofibrosis or polycythemia vera, but not typical of primary immune thrombocytopenia (Figure 2). However, not all features were present in all cases, and statistical significance was not reached in our small sample (Table 3). The myeloid to erythroid ratio and other megakaryocytic morphological features (such as the presence of maturational defects or the presence of bulbous and naked nuclei) did not show a significant difference between the pre-therapy and the on-therapy examinations.

\section{Fibrosis Evaluation}

Four of eight patients (50\%) showed a slight increase in reticulin fibrosis from MF-0 at baseline to MF-1. The remaining four patients showed the same degree of fibrosis in both biopsies: two patients were graded as MF-0, one MF-1 and one MF-2 (Table 4). The mean duration of exposure to thrombopoietin receptor agonists to the on-therapy biopsy was 2.7 years (range: 0.2-6.1 years). The increase in fibrosis content was statistically significant $(P=0.04)$. However, trichrome staining did not reveal deposition of mature collagen in any of the biopsies (Figure 1).

\section{Evaluation of Immunostaining for Extracellular Matrix Components and Stromal Cells}

The immunohistochemical investigation of extracellular matrix and stromal cells markers did not highlight any significant differences between baseline and on-therapy samples. Immunostaining for laminin and collagen IV was negative in all patients before therapy. The expression of laminin was focally positive in the on-therapy biopsy from one patient, whereas collagen IV remained negative in all biopsies. Smooth muscle actin paralleled the expression of laminin: none of the cases showed smooth muscle actin-positive cells before therapy, and only one showed a focal positivity after being on therapy. Stromal cells' low-affinity nerve growth factor receptor expression before therapy varied from focally positive to strongly positive in all the evaluable cases; in the second marrow evaluation, low-affinity nerve growth factor receptor expression was the same as before therapy in most cases (it increased and decreased in one case each). The

Table 1 Summary of patients' clinical data

\begin{tabular}{|c|c|c|c|c|c|c|c|c|c|}
\hline$P t$ & Sex & $\begin{array}{l}\text { Age: } \\
\text { pre-tx } \\
\text { (years) }\end{array}$ & $\begin{array}{c}\text { Age: } \\
\text { on-tx } \\
\text { (years) }\end{array}$ & $\begin{array}{c}\text { Time from } \\
\text { start of tx to } \\
\text { baseline } b x \\
\text { (years) }\end{array}$ & $\begin{array}{c}\text { Time from } \\
\text { start of } t x \\
\text { to on-tx } b x \\
\text { (years) }\end{array}$ & $\begin{array}{c}\text { Total } \\
\text { follow-up } \\
\text { (years) }\end{array}$ & $\begin{array}{c}\text { PLT: pre-tx } \\
\left(\times 10^{9} / 1\right)\end{array}$ & $\begin{array}{c}\text { PLT: on-tx } \\
\left(\times 10^{9} / 1\right)\end{array}$ & Splenectomy \\
\hline 1 & $\mathrm{M}$ & 56 & 58 & 1 & 1.13 & 2.13 & 7 & 30 & Yes \\
\hline 2 & $\mathrm{~F}$ & 65 & 71 & 1.34 & 4.68 & 6.02 & 24 & 272 & Yes \\
\hline 3 & $\mathrm{~F}$ & 49 & 54 & 1.91 & 3.99 & 5.91 & 26 & 20 & No \\
\hline 4 & $\mathrm{~F}$ & 70 & 72 & 0.52 & 2.36 & 2.87 & 17 & 34 & Yes \\
\hline 5 & $\mathrm{~F}$ & 43 & 44 & 0.5 & 0.21 & 0.71 & 17 & 144 & Yes \\
\hline 6 & $\mathrm{M}$ & 15 & 19 & 1.71 & 1.84 & 3.55 & 17 & 174 & Yes \\
\hline 7 & $\mathrm{~F}$ & 15 & 18 & 1.9 & 1.34 & 3.24 & 12 & 36 & Yes \\
\hline \multirow[t]{3}{*}{8} & $\mathrm{M}$ & 5 & 13 & 1.78 & 6.17 & 7.95 & 22 & 134 & No \\
\hline & Ratio & Mean & Mean & Mean & Mean & Mean & Mean & Mean & Yes/no \\
\hline & $\mathrm{M} / \mathrm{F}=0.6$ & 39.7 & 43.6 & 1.3 & 2.7 & 4 & 17.7 & 105.5 & $6 / 2$ \\
\hline
\end{tabular}

Abbreviations: baseline bx: bone marrow biopsy performed before therapy; on-tx: during therapy; on-tx bx: bone marrow biopsy performed during therapy; Pt: patient; pre-tx: before therapy; PLT: platelets; yrs: years.

Figure 1 Bone marrow morphological features before and during therapy with thrombopoietin receptor agonists. Bone marrow biopsies from patient 3 before thrombopoietin receptor agonists treatment (baseline) and during treatment. Sections were stained with hematoxylin and eosin (H\&E), reticulin and trichrome stains as indicated. The on-treatment biopsy showed increase in cellularity (b; $\times 20$ magnification) in comparison with baseline biopsy $(\mathbf{a} ; \times 20$ magnification); megakaryocytes were increased in number and formed loose clusters in the on-treatment sample $(\mathbf{d} ; \times 40$ magnification) in comparison with baseline (c; $\times 40$ magnification). Reticulin increased from MF-0 at baseline to MF-1 during the therapy (e and f; both $\times 40$ magnification). No collagen fibrosis (trichrome stain) was detected in either the baseline (g; $\times 20$ magnification) or the on-treatment samples $(\mathbf{h} ; \times 20$ magnification). Blasts were not increased at any time. 
Pre-treatment biopsy
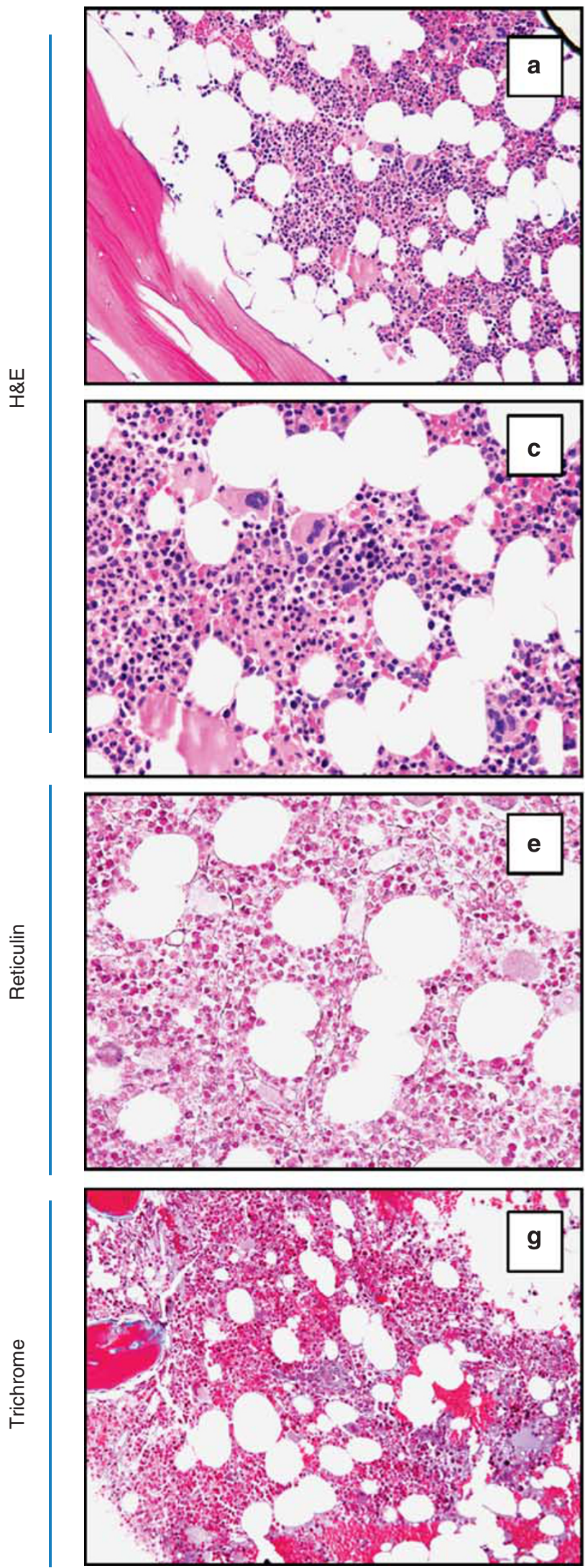

On-treatment biopsy
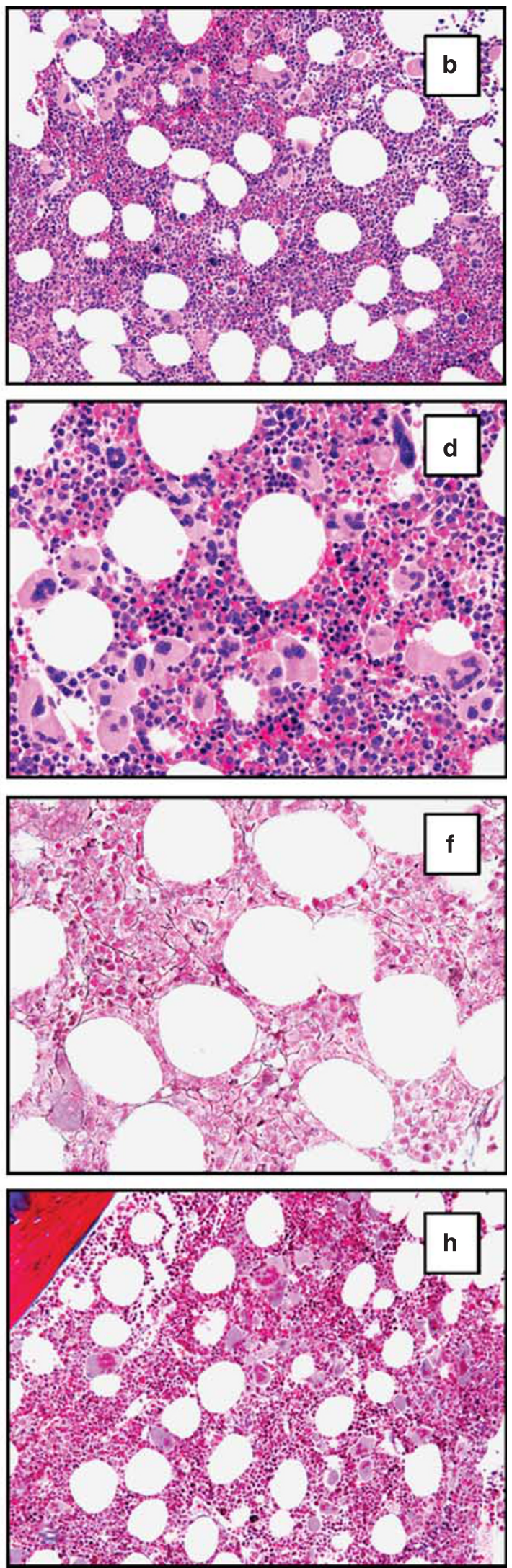
Table 2 Summary of bone marrow morphological features including bone marrow cellularity, myeloid/erythroid ratio and number of megakaryocytes, before and during therapy with thrombopoietin receptor agonists

\begin{tabular}{|c|c|c|c|c|c|c|c|c|c|c|}
\hline$P t$ & $\begin{array}{l}\text { Age: } \\
\text { pre-tx } \\
\text { (yrs) }\end{array}$ & $\begin{array}{l}\text { Age: } \\
\text { on-tx } \\
\text { (yrs) }\end{array}$ & $\begin{array}{c}B M \\
\text { cellularity: } \\
\text { pre-tx (\%) }\end{array}$ & $\begin{array}{c}B M \\
\text { cellularity: } \\
\text { on-tx (\%) }\end{array}$ & $\begin{array}{l}\text { Age adj } \\
\text { BM cellularity: } \\
\text { pre-tx }\end{array}$ & $\begin{array}{l}\text { Age adj BM } \\
\text { cellularity: } \\
\text { on-tx }\end{array}$ & $\begin{array}{l}M / E \\
\text { ratio: } \\
\text { pre-tx }\end{array}$ & $\begin{array}{l}M / E \\
\text { ratio: } \\
\text { on-tx }\end{array}$ & $\begin{array}{l}\text { Number } \\
\text { of } M K s: \\
\text { pre-tx }\end{array}$ & $\begin{array}{c}\text { Number } \\
\text { of } M K s: \\
\text { on-tx }\end{array}$ \\
\hline 1 & 56 & 58 & 40 & 70 & Normocellular & Hypercellular & 3 & 3 & $1+$ & $2+$ \\
\hline 2 & 65 & 71 & 40 & 50 & Normocellular & Hypercellular & 2 & 2 & $1+$ & $3+$ \\
\hline 3 & 49 & 54 & 50 & 70 & Normocellular & Hypercellular & 2 & 3 & $1+$ & $3+$ \\
\hline 4 & 70 & 72 & 60 & 80 & Hypercellular & Markedly hypercellular & 2 & 5 & $2+$ & $3+$ \\
\hline 5 & 43 & 44 & 60 & 70 & Normocellular & Hypercellular & 2 & 2 & $2+$ & $3+$ \\
\hline 6 & 15 & 19 & 50 & 70 & Hypocellular & Normocellular & 2 & 2 & $3+$ & $3+$ \\
\hline 7 & 15 & 18 & 80 & 90 & Normocellular & Hypercellular & 2 & 2 & $3+$ & $3+$ \\
\hline \multirow[t]{3}{*}{8} & 5 & 13 & 90 & 80 & Normocellular & Normocellular & 2 & 2 & $3+$ & $3+$ \\
\hline & Mean & Mean & Mean & Mean & & & Mean & Mean & & \\
\hline & 39.7 & 43.6 & 60 & 70 & & & 2.1 & 2.6 & & \\
\hline
\end{tabular}

Abbreviations: age adj cellularity: age adjusted cellularity; BM: bone marrow; M/E ratio: myeloid/erythroid ratio; MKs: megakaryocytes; on-tx: during therapy; Pt: patient; pre-tx: before therapy.

0 , normal; 1+, slightly increased; 2+, moderate increase; 3+, marked increase.

Case 3

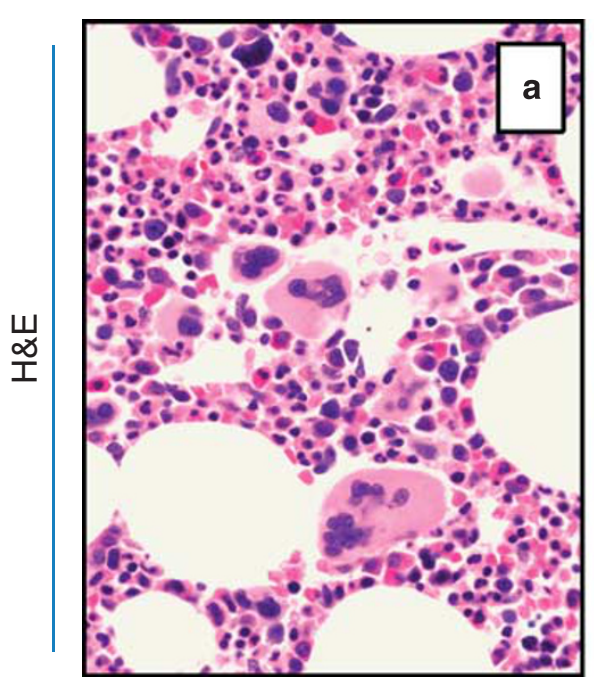

Case 5

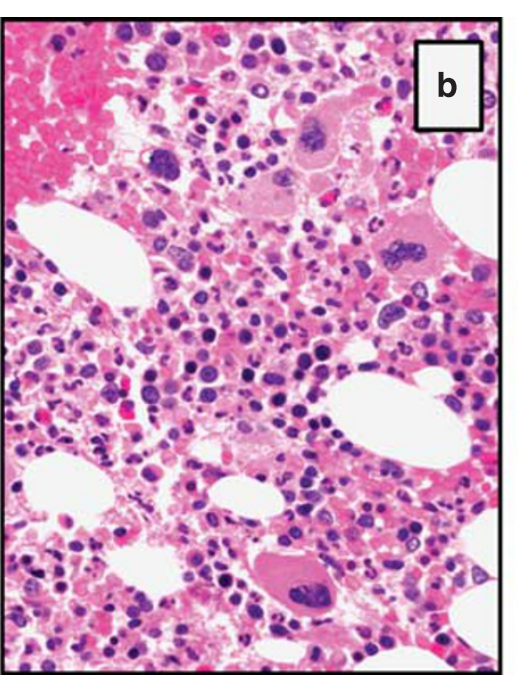

Case 7

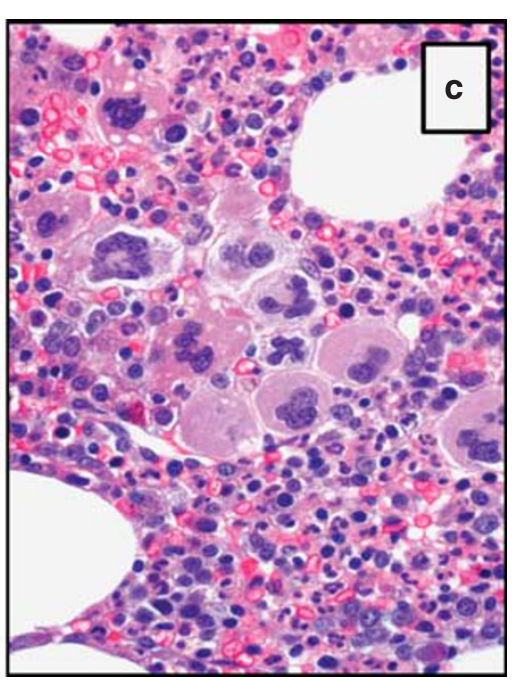

Figure 2 Megakaryocytic alterations observed in course of treatment with thrombopoietin receptor agonists. H\&E sections of on-therapy bone marrow biopsies from patient 3 (a), 5 (b) and 7 (c). Megakaryocytes showed some myeloproliferative neoplasm-like features, including a marked degree of pleomorphism with an increased proportion of large cells displaying nuclear hyperlobulation and/or abnormally condensed chromatin; there was also a tendency of megakaryocytes to form clusters (c).

Table 3 Results of the evaluation of megakaryocytic features before and during therapy with thrombopoietin receptor agonists

\begin{tabular}{|c|c|c|c|c|c|c|c|c|c|c|c|c|}
\hline$P t$ & $\begin{array}{c}\text { Pleomorphic } \\
\text { MKs: } \\
\text { pre-tx }\end{array}$ & $\begin{array}{l}\text { Pleomorphic } \\
\text { MKs: on-tx }\end{array}$ & $\begin{array}{l}\text { Clusters: } \\
\text { pre-tx }\end{array}$ & $\begin{array}{l}\text { Clusters: } \\
\text { on-tx }\end{array}$ & $\begin{array}{c}\text { Maturation } \\
\text { defects: } \\
\text { pre-tx }\end{array}$ & $\begin{array}{l}\text { Maturation } \\
\text { defects: } \\
\text { on-tx }\end{array}$ & $\begin{array}{c}\text { Hyperlobated } \\
\text { nuclei: } \\
\text { pre-tx }\end{array}$ & $\begin{array}{l}\text { Hyperlobated } \\
\text { nuclei: } \\
\text { on-tx }\end{array}$ & $\begin{array}{l}\text { Bulbous } \\
\text { nuclei: } \\
\text { pre-tx }\end{array}$ & $\begin{array}{l}\text { Bulbous } \\
\text { nuclei: } \\
\text { on-tx }\end{array}$ & $\begin{array}{l}\text { Naked } \\
\text { nuclei: } \\
\text { pre-tx }\end{array}$ & $\begin{array}{l}\text { Naked } \\
\text { nuclei: } \\
\text { on-tx }\end{array}$ \\
\hline 1 & Yes & Yes & No & Yes & No & No & No & Yes & No & No & Yes & Yes \\
\hline 2 & No & Yes & No & Yes & No & Yes & No & Yes & No & No & Yes & Yes \\
\hline 3 & Yes & Yes & No & Yes & Yes & Yes & No & Yes & No & No & Yes & Yes \\
\hline 4 & Yes & Yes & No & Yes & No & Yes & No & No & Yes & Yes & Yes & No \\
\hline 5 & No & Yes & No & Yes & No & Yes & No & Yes & No & No & Yes & Yes \\
\hline 6 & Yes & Yes & Yes & Yes & Yes & Yes & No & Yes & Yes & Yes & Yes & Yes \\
\hline 7 & No & Yes & Yes & Yes & Yes & No & Yes & Yes & No & Yes & Yes & Yes \\
\hline 8 & Yes & Yes & Yes & Yes & No & No & No & Yes & No & No & Yes & Yes \\
\hline
\end{tabular}

Abbreviations: MKs: megakaryocytes; on-tx: during therapy; Pt: patient; pre-tx: before therapy. 
Table 4 Grading of fibrosis and immunohistochemical evaluations of the extracellular matrix, before and during therapy

\begin{tabular}{|c|c|c|c|c|c|c|c|c|c|c|}
\hline$P t$ & $\begin{array}{l}\text { Fibrosis: } \\
\text { pre-tx }\end{array}$ & $\begin{array}{l}\text { Fibrosis: } \\
\text { on-tx }\end{array}$ & $\begin{array}{l}\text { Collagen } \\
I V: \text { pre-tx }\end{array}$ & $\begin{array}{l}\text { Collagen } \\
\text { IV: on-tx }\end{array}$ & $\begin{array}{l}\text { Laminin: } \\
\text { pre-tx }\end{array}$ & $\begin{array}{l}\text { Laminin: } \\
\text { on-tx }\end{array}$ & $\begin{array}{l}\text { SMA: } \\
\text { pre-tx }\end{array}$ & $\begin{array}{l}\text { SMA: } \\
\text { on-tx }\end{array}$ & $\begin{array}{l}\text { LNGFR: } \\
\text { pre-tx }\end{array}$ & $\begin{array}{c}\text { LNGFR: } \\
\text { on-tx }\end{array}$ \\
\hline 1 & MF-0 & MF-0 & NA & NA & NA & NA & NA & NA & NA & NA \\
\hline 2 & MF-0 & MF-1 & $(-)$ & $(-)$ & $(-)$ & $(-)$ & $(-)$ & $(-)$ & $1+$ & $1+$ \\
\hline 3 & MF-0 & MF-1 & $(-)$ & $(-)$ & $(-)$ & $(-)$ & $(-)$ & $1+$ & $3+$ & $3+$ \\
\hline 4 & MF-2 & MF-2 & NA & $(-)$ & NA & $(-)$ & NA & $(-)$ & NA & $2+$ \\
\hline 5 & MF-1 & MF-1 & $(-)$ & $(-)$ & $(-)$ & $1+$ & $(-)$ & $(-)$ & $2+$ & $3+$ \\
\hline 6 & MF-0 & MF-1 & $(-)$ & $(-)$ & $(-)$ & $(-)$ & $(-)$ & $(-)$ & $1+$ & $1+$ \\
\hline 7 & MF-0 & MF-1 & $(-)$ & $(-)$ & $(-)$ & $(-)$ & $(-)$ & $(-)$ & $2+$ & $(-)$ \\
\hline 8 & MF-0 & MF-0 & $(-)$ & $(-)$ & $(-)$ & $(-)$ & $(-)$ & $(-)$ & $1+$ & $1+$ \\
\hline
\end{tabular}

Abbreviations: LNGFR: low-affinity nerve growth factor receptor; NA: not available; on-tx: during therapy; Pt: patient; pre-tx: before therapy; SMA: smooth muscle actin; yrs: years.

(-), negative; 1+, focally positive, 2+, positive, 3+, strongly, diffusely positive.

expression of immunohistochemical markers did not correlate with reticulin staining. Detailed results for the evaluation of reticulin, laminin, collagen IV, smooth muscle actin and low-affinity nerve growth factor receptor are summarised in Table 4.

\section{Discussion}

Recently, concerns have been raised regarding the possibility of a fibrogenic effect of thrombopoietin receptor agonists on the bone marrow of primary immune thrombocytopenia patients receiving longterm treatment with these drugs,${ }^{12}$ but very limited published information is available. Our study represents the largest series of patients with primary immune thrombocytopenia receiving thrombopoietin receptor agonists, with a long follow-up after the start of therapy in which pre- and on-therapy bone marrow samples were examined for thrombopoietin receptor agonists-related changes. In addition, it provides the most detailed description available so far of the morphology and immunohistological features observed in the bone marrow of these patients.

Although some animal-based studies have addressed marrow effects of thrombopoietin stimulation, ${ }^{19-22}$ there is only one prior study published by Kuter et $a l^{13}$ that focused specifically on the effects of thrombopoietin receptor agonists on human marrow. In preclinical studies most data were derived from mice, which were given dosages of thrombopoietin receptor agonists much higher than those used to treat patients in clinical settings. Moreover, the short observation interval utilised in those studies has prevented a direct translation of those results to patients with primary immune thrombocytopenia. In addition, fibrosis in mice marrow was evaluated only by examining H\&E sections, without Gomori's silver staining, making those evaluations unreliable, especially when trying to distinguish between slightly different grades of fibrosis. In the only human study published so far although bone marrow biopsies were available for 10 patients, a comparison of pre-therapy and on-therapy biopsies was possible for only five patients. ${ }^{13}$

For a correct interpretation of marrow fibrosis as a possible effect of treatment, knowledge regarding normal content of stromal fibres in human marrow is warranted. Only two studies have addressed the normal distribution of reticulin and collagen fibres in the marrow of healthy individuals..$^{23,24}$ The first described a series of sternal marrow aspirates and surgical specimens, the latter a series of iliac spine biopsies. Both concur that more than $70 \%$ of healthy individuals can show a certain amount of reticulin fibrosis (up to grade 2 of the Bauermeister's scale which corresponds to MF-1 grade of the European Consensus Method). Collagen outside of vessel walls was never found in normal marrow. Recently, another study addressed specifically the issue of marrow fibrosis in a large population of primary immune thrombocytopenia patients never treated with thrombopoietin receptor agonists; patients with primary immune thrombocytopenia were showed to have a comparable degree and incidence of marrow fibrosis to that found in healthy individuals. ${ }^{25}$

In this study, we observed a slight but significant increase in reticulin fibres in half of our cases, with four of eight patients showing a one degree increase, from MF-0 to MF-1 after the initiation of therapy with thrombopoietin receptor agonists. These results are comparable to those seen by Kuter et $a l^{13}$ and seem to support the hypothesis that thrombopoietin receptor agonists can induce mild reticulin fibrosis. No instance of significant, i.e. MF-2 or MF-3 fibrosis or evidence of collagen by trichrome stain, was observed after a mean followup time of more than 2 years. These patients were selected among the good responders to thrombopoietin receptor agonists treatment; patients who did not respond would not have continued this regimen for such a long time. Even if this could represent a selection bias in our series, it gave us the possibility to observe the evolution of bone marrow stroma over the longest follow-up reported to date. 
In summary, the prevalent MF-1 fibrosis observed in the on-therapy biopsies in our series, although it represents an increase in comparison with baseline, is still compatible with normal marrow function and does not appear to be clinically relevant. ${ }^{23,24,26}$

The precise reasons and the pathogenetic mechanisms responsible for the mild fibrogenic effect exerted by thrombopoietin receptor agonists are still unknown. Increased megakaryocytic proliferation, both in experimental models and in other myelofibrotic conditions, has been associated with increased secretion of TGF- $\beta$ and other fibrogenic cytokines, which are thought capable of causing fibroblastic proliferation and extracellular matrix deposition both of reticulin and collagen. ${ }^{27,28}$ Thrombopoietin receptor agonists, by activating c-MPL and causing megakaryocytic proliferation, may lead to an increased secretion of such cytokines by the megakaryocytes into the surrounding hematopoietic stroma.

In addition to the assessment of reticulin and collagen by special stains, immunohistology was used for the first time to investigate the possibility that thrombopoietin receptor agonists could induce abnormalities in the marrow stromal composition. Overexpression of laminin, collagen IV, smooth muscle actin and nerve growth factor receptor has been associated with the presence of increased fibrosis in myelofibrotic myeloproliferative neoplasms as well as in other marrow malignancies. ${ }^{29-31}$ The lack of significant abnormalities in the expression of these antigens in the bone marrow stroma of our patients is consistent with the presence of only a mild fibrogenic process, most likely fully reversible, as previously shown by others. ${ }^{13}$

As compared with the pre-treatment biopsies, in the on-treatment samples we observed an increase in bone marrow cellularity. This was true not only when the cellularity was evaluated as a percentage but also when it was scored as decreased, increased or normocellular in relationship with the age expected value. Hypercellularity was due to increased hematopoiesis in all three main hematopoietic lineages. In the majority of cases megakaryocytes showed some myeloproliferative neoplasm-like features, including a marked degree of pleomorphism with an increased proportion of large cells displaying nuclear hyperlobulation and/ or abnormally condensed chromatin. There was also a tendency of megakaryocytes to form clusters, a finding which can be seen both in ET and in other MPNs such as primary myelofibrosis but is not seen normally.

The increase in cellularity and the acquisition of myeloproliferative neoplasm-like features are novel findings. Their presence suggests that thrombopoietin administration may exert pleiotropic effects on human bone marrow. The thrombopoietin receptor c-MPL, a member of the super-family of cytokine receptors, has a dimeric structure with tyrosine kinase intracellular domains, and activates different downstream pathways involving JAK-2, IP3 and RAS. ${ }^{32}$ The down-stream effects are the proliferation, differentiation and maturation of megakaryocytes. In addition, c-MPL is present on hematopoietic stem cells. ${ }^{33-38}$

Several studies have demonstrated that thrombopoietin or thrombopoietin receptor agonists could stimulate stem cells proliferation in vitro before bone marrow transplant. ${ }^{35,36}$ Small molecule agonists of c-MPL can stimulate stem cells to differentiate not only to megakaryocytes, but also towards erythroid and myeloid precursors. ${ }^{35,37}$ Thrombopoietin or thrombopoietin receptor agonists, and c-MPL are part of a complex network of cytokines and receptors, whose delicate balance is crucial in the tuning of the process of hematopoietic differentiation. ${ }^{34,36,37,39,40}$ The increased hematopoiesis seen in treated patients could be related to the effects of thrombopoietin receptor agonists on the stem cell reservoir or on early progenitors of each lineage. c-MPL activation effects are mediated by the phosphorylation of JAK2: this protein kinase is often mutated in $\mathrm{MPNs}^{41}$ and it is thought to have a major role in the pathogenesis of these diseases, even if the exact molecular mechanisms are still unclear. ${ }^{32}$ It is intriguing that the marrow of some primary immune thrombocytopenia patients treated with thrombopoietin receptor agonists shows myeloproliferativelike features, a finding which supports the hypothesis of a partial overlap with MPNs in terms of the molecular pathways involved. No chromosomal or molecular abnormalities were found in the primary immune thrombocytopenia patients enrolled in this study with cytogenetic and FISH analysis. Therefore the possibility of transformation to a clonal myeloproliferative disease seems very unlikely and the morphological changes observed appear to be purely reactive. However, further studies would be needed to shed light on the exact mechanism leading to the morphological changes observed in primary immune thrombocytopenia patients treated with thrombopoietin receptor agonists. In addition, as thrombopoietin receptor agonists become a mainstream tool in the therapy for patients with primary immune thrombocytopenia, general pathologists and hematopathologists need to be aware of the characteristic morphological features associated with these drugs, helping avoid misdiagnosis of malignancy.

In conclusion, this is the first study with a detailed description of the characteristic morphological changes observed in the bone marrow of primary immune thrombocytopenia patients treated with thrombopoietin receptor agonists. We have observed a mild increase in reticulin fibres in half of the patients treated consistently for more than 2 years; however, this increase never reached clinically significant levels after 2 years of followup. We also observed myeloproliferative-like changes during the therapy, including an increase in cellularity and megakaryocytic hyperplasia and 
atypia. Further studies will be helpful both in better characterizing the progress of fibrosis deposition after even longer follow-up intervals in patients treated with thrombopoietin receptor agonists and in shedding light on the pathobiology of myeloproliferative neoplasms.

\section{Disclosure/conflict of interest}

LB, AO, MA and JG declare no conflict of interest. WG has received research grants from Amgen, and received honorariums from Amgen and GlaxoSmithKline (GSK), whose products were studied in the present report. JB currently receives clinical research support from Amgen, Cangene, GlaxoSmithKline, Genzyme, IgG of America, Immunomedics, Ligand, Eisai, Shionogi and Sysmex and he has participated in Advisory Boards for Amgen, GlaxoSmithKline, Ligand, Shionogi and Eisai; he also had a 1 day consult with Portola and his family owns stock in Amgen and GlaxoSmithKline.

\section{References}

1 Rodeghiero F, Stasi R, Gernsheimer T, et al. Standardization of terminology, definitions and outcome criteria in immune thrombocytopenic purpura of adults and children: report from an international working group. Blood 2009;113:2386-2393.

2 Cines DB, Bussel JB. How I treat idiopathic thrombocytopenic purpura (ITP). Blood 2005;106:2244-2251.

3 Bussel JB. Traditional and new approaches to the management of immune thrombocytopenia: issues of when and who to treat. Hematol Oncol Clin North Am 2009;23:1329-1341.

4 Hasan A, Michel M, Patel V, et al. Repeated courses of rituximab in chronic ITP: three different regimens. Am J Hematol 2009;84:661-665.

5 Aleem A, Alaskar AS, Algahtani F, et al. Rituximab in immune thrombocytopenia: transient responses, low rate of sustained remissions and poor response to further therapy in refractory patients. Int J Hematol 2010;92:283-288.

6 Wang J, Wiley JM, Luddy R, et al. Chronic immune thrombocytopenic purpura in children: assessment of rituximab treatment. J Pediatr 2005;146:217-221.

7 Bussel JB, Kuter DJ, George JN, et al. AMG 531, a thrombopoiesis-stimulating protein, for chronic ITP. N Engl J Med 2006;355:1672-1681.

8 Bussel JB, Cheng G, Saleh MN, et al. Eltrombopag for the treatment of chronic idiopathic thrombocytopenic purpura. N Engl J Med 2007;357:2237-2247.

9 Sattler M, Durstin MA, Frank DA, et al. The thrombopoietin receptor c-MPL activates JAK2 and TYK2 tyrosine kinases. Exp Hematol 1995;23:1040-1048.

10 Rojnuckarin P, Drachman JG, Kaushansky K. Thrombopoietin-induced activation of the mitogen-activated protein kinase (MAPK) pathway in normal megakaryocytes: role in endomitosis. Blood 1999;94: 1273-1282.

11 Miyakawa Y, Rojnuckarin P, Habib T, et al. Thrombopoietin induces phosphoinositol 3-kinase activation through SHP2, Gab, and insulin receptor substrate proteins in BAF3 cells and primary murine megakaryocytes. J Biol Chem 2001;276:2494-2502.

12 Bussel JB, Kuter DJ, Pullarkat V, et al. Safety and efficacy of long-term treatment with romiplostim in thrombocytopenic patients with chronic ITP. Blood 2009;113:2161-2171.

13 Kuter DJ, Mufti GJ, Bain BJ, et al. Evaluation of bone marrow reticulin formation in chronic immune thrombocytopenia patients treated with romiplostim. Blood 2009;114:3748-3756.

14 Chiu A, Nanaji NM, Czader M, et al. The stromal composition of mast cell aggregates in systemic mastocytosis. Mod Pathol 2009;22:857-865.

15 Thiele J, Kvasnicka HM, Diehl V. Standardization of bone marrow features-does it work in hematopathology for histological discrimination of different disease patterns? Histol Histopathol 2005;20:633-644.

16 Douglas VK, Tallman MS, Cripe LD, et al. Thrombopoietin administered during induction chemotherapy to patients with acute myeloid leukemia induces transient morphologic changes that may resemble chronic myeloproliferative disorders. Am J Clin Pathol 2002;117:844-850.

17 Gianelli U, Iurlo A, Vener C, et al. The significance of bone marrow biopsy and JAK2V617F mutation in the differential diagnosis between the 'early' prepolycythemic phase of polycythemia vera and essential thrombocythemia. Am J Clin Pathol 2008;130:336-342.

18 Thiele J, Kvasnicka HM, Facchetti F, et al. European consensus on grading bone marrow fibrosis and assessment of cellularity. Haematologica 2005;90:1128-1132.

19 Yan XQ, Lacey D, Hill D, et al. A model of myelofibrosis and osteosclerosis in mice induced by overexpressing thrombopoietin (mpl ligand): reversal of disease by bone marrow transplantation. Blood 1996;88:402-409.

20 Villeval JL, Cohen-Solal K, Tulliez M, et al. High thrombopoietin production by hematopoietic cells induces a fatal myeloproliferative syndrome in mice. Blood 1997;90:4369-4383.

21 Frey BM, Rafii S, Teterson M, et al. Adenovectormediated expression of human thrombopoietin cDNA in immune-compromised mice: insights into the pathophysiology of osteomyelofibrosis. J Immunol 1998;160:691-699.

22 Yan XQ, Lacey D, Fletcher F, et al. Chronic exposure to retroviral vector encoded MGDF (mpl-ligand) induces lineage-specific growth and differentiation of megakaryocytes in mice. Blood 1995;86:4025-4033.

23 Beckman EN, Brown Jr AW. Normal reticulin level in iliac bone marrow. Arch Pathol Lab Med 1990;114: 1241-1243.

24 Bauermeister DE. Quantitation of bone marrow reticulin-a normal range. Am J Clin Pathol 1971;56: 24-31.

25 Ettrup MS, Jensen AO, Engebjerg MC, et al. Bone marrow reticulin and collagen content in patients with adult chronic immune thrombocytopenic purpura: A Danish nationwide study. Am J Hematol 2010; 85:930-934.

26 Kuter DJ, Bain B, Mufti G, et al. Bone marrow fibrosis: pathophysiology and clinical significance of increased bone marrow stromal fibres. Br J Haematol 2007;139: 351-362.

27 Chagraoui H, Komura E, Tulliez M, et al. Prominent role of TGF-beta 1 in thrombopoietin-induced myelofibrosis in mice. Blood 2002;100:3495-3503. 
28 Ciurea SO, Merchant D, Mahmud N, et al. Pivotal contributions of megakaryocytes to the biology of idiopathic myelofibrosis. Blood 2007;110: 986-993.

29 Thiele J, Rompcik V, Wagner S, et al. Vascular architecture and collagen type IV in primary myelofibrosis and polycythaemia vera: an immunomorphometric study on trephine biopsies of the bone marrow. Br J Haematol 1992;80:227-234.

30 Lisse I, Hasselbalch H, Junker P. Bone marrow stroma in idiopathic myelofibrosis and other haematological diseases. An immunohistochemical study. APMIS 1991;99:171-178.

31 Caneva L, Soligo D, Cattoretti G, et al. Immunoelectron microscopy characterization of human bone marrow stromal cells with anti-NGFR antibodies. Blood Cells Mol Dis 1995;21:73-85.

32 Levine RL, Pardanani A, Tefferi A, et al. Role of JAK2 in the pathogenesis and therapy of myeloproliferative disorders. Nat Rev Cancer 2007;7:673-683.

33 Kaushansky K, Lok S, Holly RD, et al. Promotion of megakaryocyte progenitor expansion and differentiation by the c-Mpl ligand thrombopoietin. Nature 1994;369:568-571.

34 Alexander WS, Roberts AW, Nicola NA, et al. Deficiencies in progenitor cells of multiple hematopoietic lineages and defective megakaryocytopoiesis in mice lacking the thrombopoietic receptor c-Mpl. Blood 1996;87:2162-2170.

35 Sitnicka E, Lin N, Priestley GV, et al. The effect of thrombopoietin on the proliferation and differentiation of murine hematopoietic stem cells. Blood 1996;87: 4998-5005.

36 Nishino T, Miyaji K, Ishiwata N, et al. Ex vivo expansion of human hematopoietic stem cells by a small-molecule agonist of c-MPL. Exp Hematol 2009;37:1364-1377 e4.

37 Qian H, Buza-Vidas N, Hyland CD, et al. Critical role of thrombopoietin in maintaining adult quiescent hematopoietic stem cells. Cell Stem Cell 2007;1:671-684.

38 Kaushansky K. Thrombopoietin: accumulating evidence for an important biological effect on the hematopoietic stem cell. Ann NY Acad Sci 2003;996:39-43.

39 Yamazaki S, Nakauchi H. Insights into signaling and function of hematopoietic stem cells at the single-cell level. Curr Opin Hematol 2009;16:255-258.

40 Yoshihara H, Arai F, Hosokawa K, et al. Thrombopoietin/MPL signaling regulates hematopoietic stem cell quiescence and interaction with the osteoblastic niche. Cell Stem Cell 2007;1:685-697.

41 Levine RL, Wadleigh M, Cools J, et al. Activating mutation in the tyrosine kinase JAK2 in polycythemia vera, essential thrombocythemia, and myeloid metaplasia with myelofibrosis. Cancer Cell 2005;7:387-397. 